\title{
THE WEAKLY CONTAGIOUS STOCHASTIC PROCESS WHICH DEPENDS UPON THE GAUSSIAN DISTRIBUTION
}

\author{
By
}

\section{Tosio KITAGaWA}

(Received January 16, 1941)

\$1. Introduction. The object of this paper is to introduce a perhaps new scheme of the weakly contagious stochastic process which is defined over a continuous parameter and which depends upon the normal (gaussian) distribution in a certain sense. Our scheme might be also recognized to be a continuous analogy of the well-known one due to PólyA-EgGenberger $\left({ }^{1}\right)$. The original Pólya-EgGenberger's scheme is defined in the following way. Let us consider a bag containing $N$ balls, where $R$ balls are white and $S$ ones black, $N$ being equal to $R+S$. We shall pick a ball out of this bag at random, and return back $1+\Delta$ balls of the same colour with that of the picked one. The probability that we shall have $k$ white balls in the $n$ trials is given by

$$
\left(\begin{array}{l}
n \\
k
\end{array}\right) \frac{R(R+\Delta) \ldots(R+(k-1) d) S(S+\Delta) \ldots(S+(n-k-1) \Delta)}{N(N+\Delta)(N+2 \Delta) \ldots(N+(n-1) d)} .
$$

If $\Delta$ is positive, the number of the balls of the bag increases after each trial, each success favours the chances of the success in the future, each failure damages them, and hence the scheme is contagious. For the more general formulation we found it

(1) F. Eggenberger and G. Pólya: Ueber die Statistik verketteter Vorgänge, Z. f. angew. Math. u. Mechanik, 3 (1923), 276-289.

G. Pólya: Sur quelques points de la théorie des probabilités, Ann. Inst. Henri Poincaré, 1 (1931), 117-161. 
necessary to generalise this scheme $\left({ }^{2}\right)$. Here we shall repeat some definitions, which were introduced in a previous paper, in a slightly modified form so far as they are necessary for the following argument.

Definition 1. A sequence of the stochastic variables $\mathrm{Y}_{1}, \mathrm{Y}_{2}, \ldots$, $\mathrm{Y}_{n}, \ldots$ is said to be contagious with a sequence of the generating distribution functions (g. d. $f).\left\{F_{k}(x)\right\}(k=1,2,3, \ldots)$ and a contagion-parameter $\delta$, if the following conditions are fulfilled.

$\left(1^{\circ}\right)$ The distribution function $\left(d . f\right.$.) of $\mathrm{Y}_{1}$ is $F_{1}(x)$.

$\left(2^{\circ}\right)$ For each positive integer $r$ subject to $r \geqq 2, \mathrm{Y}_{r}$ is a stochastic variable which depends upon $\mathrm{Y}_{1}, \mathrm{Y}_{2}, \ldots, \mathrm{Y}_{r-2}$ and $\mathrm{Y}_{r-1}$ in such a manner that, for any system of $r-1$ real numbers $\left(\alpha_{1}\right.$, $\left.\alpha_{2}, \ldots, \alpha_{r-2}, \alpha_{r-1}\right)$, the circumstance that $\mathrm{Y}_{1}=a_{1}, \mathrm{Y}_{2}=\alpha_{2}, \ldots, \mathrm{Y}_{r-2}=$ $\alpha_{r-2}$ and $\mathrm{Y}_{r-1}=\alpha_{r-1}$ implies that the d.f. is given by

$$
G_{r}\left(x ; \alpha_{1}, \alpha_{2}, \ldots, \alpha_{r-1}\right)=\frac{F_{r}(x)+\delta \sum_{\nu=0}^{r-1} E\left(x-\alpha_{v}\right)}{1+(r-1) \delta},
$$

where we denote by $E(x)$ the d.f. such that $E(x)=0$ for $x<0$ and $E(x)=1$ for $x \geqq 0$.

Definition 2. A doubly infinite matrix of the stochastic variables $\left\|\mathrm{Y}_{n, k}\right\|(k=1,2,3, \ldots ; n=1,2,3, \ldots)$ is said to be an infinite matrix of the contagious stochastic variables with an infinite matrix of the g. d. $f .\left\|F_{n, k}(x)\right\|(k=1,2,3, \ldots ; n=1,2,3, \ldots)$ and with an infinite sequence of the contagion-parameters $\left\{\grave{o}_{n}\right\}$, if, for each $n,\left\{\mathrm{Y}_{n, k}\right\} \quad(k=1,2,3, \ldots)$ is the sequence of the contagious stochastic variables with the sequence of the g. $d . f$. $\left\{F_{n, k}(x)\right\} \quad(k=1,2,3, \ldots)$ and with the contagion-parameter $\grave{o}_{n}$.

Definition 3. An infinite matrix of the contagious stochastic variables with the sequence of the contagion-parameters $\left\{\hat{o}_{n}\right\} \quad(n=1$, $2,3, \ldots)$ is said to be weakly contagious, if $\hat{o}_{n} \geqq 0$ and $\lim _{n \rightarrow \infty} n \hat{o}_{n}$ exists and $=d \geqq 0$.

The idea which is fundamentally important in leading us to the continuous analogy of the PólYA-EgGenberger's scheme consists in taking into the consideration the partial sums of the

(2) T. Kitagawa: The Limit-Theorems of the Contagious Stochastic Process, Mem. Fac. Sc. Kyūsyū Imp. Univ., Ser. A, 1 (1941) 167-194. 
sequence of the stochastic variables $\left\{\mathrm{Y}_{n, k}\right\}(k=1,2,3, \ldots)$. To each assigned positive number $t$, we shall correspond a sequence of the stochastic variables

$$
\mathrm{S}_{n}\left(\frac{m_{n}}{n}\right) \equiv \sum_{k=1}^{m_{n}} \mathrm{Y}_{n, k} \quad(n=1,2,3, \ldots),
$$

where the positive integers $m_{n}$ are subject to the condition that

$$
\lim _{n \rightarrow \infty} m_{n} / n=t
$$

and we shall seek the limiting properties of the sequences of the weakly contagious stochastic variables as $n$ becomes infinity.

In this preliminary report we shall confine ourselves with a highly specialized case of the g. d. f.: we shall make the following assumption :

Assumption I. All of the g.d.f. $\left\{F_{n, k}(x)\right\}(k=1,2,3, \ldots$; $n=1,2,3, \ldots)$ coincide with a common $d . f . F(x)$ which is a step -function with jumps $\rho$ and $\sigma(=1-\rho)$ at the points $x=1$ and $x=0$ respectively.

In what follows we shall prove that, to each assigned positive number $t$, there are sequences of constants $\left\{A_{n}\right\}$ and $\left\{B_{n}\right\}$, both depending upon $t$, such that $\left\{\left\{S_{n}\left(m_{n} / n\right)-A_{n}\right\} / B_{n}\right\}(n=1,2,3, \ldots)$ converges to a certain d.f., that is, converges in the sense of the probabilistic law, and we shall then propose to introduce the random function which is defined over $(0, \infty)$ and whose probabilistic laws are governed by the limiting probabilistic laws which we shall have thus obtained. Our formulation is, however, in some respects formal, and the strict formulation and the continuity property of our process are remaining in this paper.

$\S 2$. Limiting Probabilistic Laws. Let us put

$$
\operatorname{Pr} .\left\{\mathrm{Y}_{n, 1}+\mathrm{Y}_{n, 2}+\ldots+\mathrm{Y}_{n, m_{n}}=k\right\} \equiv \operatorname{Pr} .\left\{S_{n}\left(m_{n} / n\right)=k\right\} \text {. }
$$

[1] In view of our Assumption and (1.01), we shall have

$$
\begin{aligned}
& \sum_{k=l_{1}}^{l_{2}} \operatorname{Pr} \cdot\left\{\mathrm{S}_{n}\left(m_{n} / n\right)=k\right\} \\
& =\sum_{k=l_{1}}^{l_{2}}\left(\begin{array}{c}
m_{n} \\
k
\end{array}\right) \frac{\rho\left(\rho+\grave{o}_{n}\right) \ldots\left(\rho+(k-1) \hat{o}_{n}\right) \sigma\left(\sigma+\hat{o}_{n}\right) \cdots\left(\sigma+\left(m_{n}-k-1\right) \hat{o}_{n}\right)}{\left(1+\hat{o}_{n}\right)\left(1+2 \hat{o}_{n}\right) \ldots\left(1+\left(m_{n}-1\right) \hat{o}_{n}\right)} .
\end{aligned}
$$

We shall estimate (10.1) under the hypothesis that 


$$
\left\{\begin{array}{l}
\text { (i) } m_{n}=n t+o(1), \quad o_{n}=t d / n \\
\text { (ii) } l_{i}=n \rho t+(n \rho \sigma t)^{1 / 2} x_{i} \quad(i=1,2)
\end{array}\right.
$$

as $n$ tends to infinity, where $t \geqq 0, d \geqq 0, \rho \geqq 0$ and $x_{i}\left(x_{1}<x_{2}\right)$ are arbitrarily assigned fixed numbers.

For this purpose we shall estimate the quotient

$$
\frac{\operatorname{Pr} .\left\{\mathrm{S}_{n}\left(m_{n} / n\right)=k\right\}-\operatorname{Pr} \cdot\left\{\mathrm{S}_{n}\left(m_{n} / n\right)=k-1\right\}}{\operatorname{Pr} .\left\{\mathrm{S}_{n}\left(m_{n} / n\right)=k-1\right\}} \equiv Q_{n, k},
$$

say, under our hypothesis and by substituting

$$
k=n \rho t+(n \rho \sigma t)^{1 / 2} x+o\left(n^{1 / 2}\right), \quad\left(x_{1}<x<x_{2}\right),
$$

$x$ being independent of $n$. By an elementary calculation we shall reach

$$
\lim _{n \rightarrow \infty}\left\{(n \rho \sigma t)^{1 / 2} Q_{n, k}\right\}=-\frac{x}{1+t d} .
$$

This means that the sequence of the d.f. of the stochastic variables $\left\{\left(\mathrm{S}_{n}\left(m_{n} / n\right)-n \rho t\right) /(n \rho \sigma t)^{1 / 2}\right\}(n=1,2,3, \ldots)$ converges to the d. f.

$$
\frac{1}{\{2 \pi(1+t d)\}^{1 / 2}} \int_{-\infty}^{x} \exp \left\{-\frac{s^{2}}{2(1+t d)}\right\} d s .
$$

[2] In the next step we shall consider the probability that two of the following events:

$$
l_{1} \leqq \mathrm{~S}_{n}\left(\frac{m_{n}^{(1)}}{n}\right)-\mathrm{S}_{n}(0) \leqq l_{2}, \quad s_{1} \leqq \mathrm{~S}_{n}\left(\frac{m_{n}^{(1)}+m_{n}^{(2)}}{n}\right)-\mathrm{S}_{n}\left(\frac{m_{n}^{(1)}}{n}\right) \leqq s_{2}
$$

will happen, which we denote by

$$
\operatorname{Pr}\left\{\left\{l_{1} \leqq \mathrm{~S}_{n}\left(\frac{m_{n}^{(1)}}{n}\right)-\mathrm{S}_{n}(0) \leqq l_{2}, \mathrm{~s}_{1} \leqq \mathrm{~S}_{n}\left(\frac{m_{n}^{(1)}+m_{n}^{(2)}}{n}\right)-\mathrm{S}_{n}\left(\frac{m_{n}^{(1)}}{n}\right) \leqq s_{2}\right\}\right.
$$

We observe readily that (2.07) is equal to

$$
\begin{aligned}
& \sum_{k=l_{1}}^{l_{2}}\left(\begin{array}{c}
m_{n}^{(1)} \\
k
\end{array}\right) \frac{\rho\left(\rho+\grave{o}_{n}\right) \ldots\left(\rho+(k-1) \hat{o}_{n}\right) \sigma\left(\sigma+\grave{o}_{n}\right) \ldots\left(\sigma+\left(m_{n}^{(1)}-k-1\right) \delta_{n}\right)}{\left(1+\grave{o}_{n}\right)\left(1+2 \grave{o}_{n}\right) \ldots\left(1+\left(m_{n}^{(1)}-1\right) \grave{o}_{n}\right)} \\
& \times \sum_{h=s_{1}}^{s_{2}}\left\{\left(\begin{array}{c}
m_{n}^{(2)} \\
h
\end{array}\right) \frac{\left(\rho+k \grave{o}_{n}\right) \ldots\left(\rho+(k+h-1) \grave{n}_{n}\right)}{\left(1+m_{n}^{(1)} \hat{o}_{n}\right) \ldots\left(1+\left(m_{n}^{(1)}+h-1\right) \hat{o}_{n}\right)}\right. \\
& \left.\times \frac{\left(\sigma+\left(m_{n}^{(1)}-k\right) \grave{o}_{n}\right) \ldots\left(\sigma+\left(m_{n}^{(1)}+m_{n}^{(2)}-k-h-1\right) \hat{o}_{n}\right)}{\left(1+\left(m_{n}^{(1)}+h\right) \grave{o}_{n}\right) \ldots\left(1+\left(m_{n}^{(1)}+m_{n}^{(2)}-1\right) \grave{o}_{n}\right)}\right\} .
\end{aligned}
$$


We shall now estimate (2.08) under the following hypothesis:

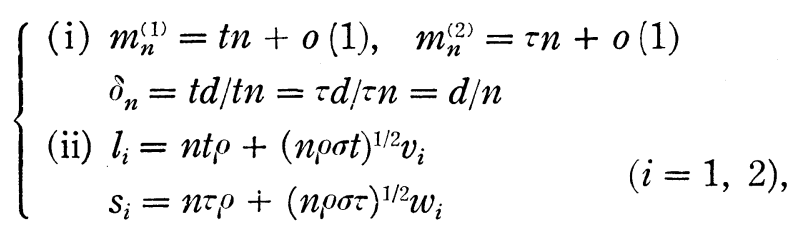

as $n$ becomes infinity, $t, \tau, d, \rho, v_{i}$ and $w_{i}$ being fixed.

For this purpose we shall put, for a moment,

$$
\begin{aligned}
q_{h}^{\left(m_{n}^{(2)}\right)}= & \left(\begin{array}{c}
m_{n}^{(2)} \\
h
\end{array}\right) \frac{\left(\rho+k \grave{o}_{n}\right) \ldots\left(\rho+(k+h-1) \grave{o}_{n}\right)}{\left(1+m_{n}^{(1)} \hat{o}_{n}\right) \ldots\left(1+\left(m_{n}^{(1)}+h-1\right) \grave{o}_{n}\right)} \\
& \times \frac{\left(\sigma+\left(m_{n}^{(1)}-k\right) \grave{\partial}_{n}\right) \ldots\left(\sigma+\left(m_{n}^{(1)}+m_{n}^{(2)}-k-h-1\right) \grave{o}_{n}\right)}{\left(1+\left(m_{n}^{(1)}+h\right) \hat{o}_{n}\right) \ldots\left(1+\left(m_{n}^{(1)}+m_{n}^{(2)}-1\right) \hat{o}_{n}\right)} .
\end{aligned}
$$

Then we have

$$
\begin{aligned}
& \frac{q_{h}^{\left(m_{n}^{(2)}\right)}-q_{h-1}^{\left(m_{n}^{(2)}\right)}}{q_{h-1}^{\left(m_{n}^{(2)}\right)}} \\
= & \frac{\left(m_{n}^{(2)}-h+1\right)\left(\rho+(k+h-1) \hat{o}_{n}\right)-h\left(\sigma+\left(m_{n}^{(1)}+m_{n}^{(2)}-k-h\right) \hat{o}_{n}\right)}{h\left\{\sigma+\left(m_{n}^{(1)}+m_{n}^{(2)}-k-h\right)_{n}\right\}} .
\end{aligned}
$$

Here we prefer to put

$$
\left\{\begin{array}{l}
h=n \rho \tau+(n \rho \sigma \tau)^{1 / 2} w+o\left(n^{1 / 2}\right) . \\
k=n \rho t+(n \rho \sigma t)^{1 / 2} v+o\left(n^{1 / 2}\right),
\end{array}\right.
$$

which gives us

$$
\begin{aligned}
\left(m_{n}^{(2)}-\right. & h+1)\left\{\rho+(k+h-1) \grave{o}_{n}\right\}-h\left\{\sigma+\left(m_{n}^{(1)}+m_{n}^{(2)}-k-h\right) \hat{o}_{n}\right\} \\
= & \left(m_{n}^{(2)}+1\right)\left\{\rho+(k-1) \grave{o}_{n}\right\}-h\left\{1+\left(m_{n}^{(1)}-2\right) \grave{o}_{n}\right\} \\
= & (n \tau+1+o(1))\left\{\rho+\frac{d}{n}\left(n \rho t+(n \rho \sigma t)^{1 / 2} v+o\left(n^{1 / 2}\right)-1\right)\right\} \\
& -\left\{n \rho \tau+(n \rho \sigma \tau)^{1 / 2} w+o\left(n^{1 / 2}\right)\right\}\left(1+(n t-2) \frac{d}{n}\right) \\
= & (n \rho \sigma \tau)^{1 / 2}\left\{(t \tau)^{1 / 2} v d-w(1+t d)\right\}+o\left(n^{1 / 2}\right),
\end{aligned}
$$

and similarly

$$
h\left\{\left(m_{n}^{(1)}+m_{n}^{(2)}\right) \hat{o}_{n}+\sigma-(k+h) \grave{\delta}_{n}\right\}
$$




$$
\begin{aligned}
& =\left\{n_{\imath} \tau+(n \rho \sigma \tau)^{1 / 2} w\right\}\left\{n(t+\tau) \frac{d}{n}+\sigma-\left(n \rho \tau+n \rho t+O\left(n^{1 / 2}\right)\right) \frac{d}{n}\right\} \\
& =n \rho \sigma \tau\{1+(t+\tau) d\}+O\left(n^{1 / 2}\right) .
\end{aligned}
$$

Substituting (2.13) and (2.14) into (2.11) we reach

$$
\left\{q_{h}^{\left(m_{n}^{(2)}\right)}-q_{h-1}^{\left(m_{n}^{(2)}\right)}\right\} / q_{h-1}^{\left(m_{n}^{(2)}\right)}=\frac{(t \tau)^{1 / 2} v d-(1+t d) w}{\{1+(t+\tau) d\}(n \rho \sigma \tau)^{1 / 2}}+O(1 / n)
$$

and hence

$$
(n \rho \sigma \tau)^{1 / 2} \frac{q_{h}^{\left(m_{n}^{(2)}\right)}-q_{h-1}^{\left(m_{n}^{(2)}\right)}}{q_{h-1}^{\left(m_{n}^{(2)}\right)}}=\frac{(t \tau)^{1 / 2} v d}{1+(t+\tau) d}-\frac{(1+t d) w}{1+(t+\tau) d}+O\left(\frac{1}{n^{1 / 2}}\right)
$$

This informs us that there exists a function $q(w)$, defined over $(-\infty, \infty)$, such that we have

$$
\frac{1}{q(w)} \frac{d q(w)}{d w}=\frac{(t \tau)^{1 / 2} v d}{1+(t+\tau) d}-\frac{(1+t d) w}{1+(t+\tau) d}
$$

whence

$$
q(w)=c(v) \exp \left\{\frac{(t \tau)^{1 / 2} v w d}{1+(t+\tau) d}-\frac{(1+t d) w^{2}}{2\{1+(t+\tau) d\}}\right\}
$$

where $c(v)$ should be determined by the condition

$$
\int_{-\infty}^{\infty} q(w) d w=1
$$

An elementary consideration gives us therefore

$$
c(v)=\left(\frac{1+t d}{2 \pi\{1+(t+\tau) d\}}\right)^{1 / 2} \exp \left\{-\frac{1+t d}{2\{1+(t+\tau) d\}}\left(\frac{(t \tau)^{1 / 2} v d}{1+t d}\right)^{2}\right\}
$$

The combination of (2.09), (2.16), (2.18) and (2.20) yields us consequently that for any two pairs of real numbers $u_{1}, u_{2}\left(u_{1}<u_{2}\right)$ and $w_{1}, w_{2}\left(w_{1}<w_{2}\right)$,

$$
\begin{aligned}
\lim _{n \rightarrow \infty} \operatorname{Pr}\left\{u_{1}<\frac{\mathrm{S}_{n}\left(\frac{m_{n}^{(1)}}{n}\right)-n \rho t}{(n \rho \sigma t)^{1 / 2}}<u_{2},\right. \\
\left.w_{1}<\frac{\mathrm{S}_{n}\left(\frac{m_{n}^{(1)}+m_{n}^{(2)}}{n}\right)-\mathrm{S}\left(\frac{m_{n}^{(1)}}{n}\right)-n \rho \tau}{(n \rho \sigma \tau)^{1 / 2}}<w_{2}\right\}
\end{aligned}
$$


(II) $=\frac{1}{\{2 \pi(1+t d)\}^{1 / 2}} \int_{u_{j} / t^{\prime / 2}}^{u_{2} / t^{1 / 2}}\left\{-\frac{u^{2}}{2(1+t d)}\right\} d u$

$$
\times\left\{\frac{1+t d}{2 \pi(1+(t+\tau) d)}\right\}^{1 / 2} \int_{w_{1} / z / 1 / 2}^{w_{2} / 1 / 1 / 2}\left\{\frac{-(1+t d)}{2(1+(t+\tau) d)}\left(w-\frac{(t \tau)^{1 / 2} u d}{1+t d}\right)^{2}\right\} d w,
$$

provided that

$$
m_{n}^{(1)} / n=t+o(1), \quad m_{n}^{(2)} / n=\tau+o(1) .
$$

[3] We have now no difficulty in calculating the probability that all of the events:

$$
E_{n}^{(k)}: a_{k} \leqq \mathrm{~S}_{n}\left(\sum_{\nu=1}^{k} m_{n}^{(\nu)} / n\right)-\mathrm{S}_{n}\left(\sum_{\nu=1}^{k-1} m_{n}^{(\nu)} / n\right) \leqq b_{k}
$$

$(k=1,2, \ldots, s)$ will happen and in estimating it as $n$ tends to infinity under the hypothesis that

$$
m_{n}^{(\nu)}=n t^{(\nu)}+o(1), \quad \grave{o}_{n}=d / n .
$$

Similarly as in the relation (II), we shall have

(III. I) $\lim _{n \rightarrow \infty} \operatorname{Pr} \cdot\left\{a_{1}<\frac{\mathrm{S}_{n}\left(t_{n}^{(1)}\right)-\mathrm{S}_{n}\left(t_{n}^{(0)}\right)}{\left(n_{i} \sigma \sigma t_{1}\right)^{1 / 2}}<b_{1}, \ldots\right.$,

$$
\begin{aligned}
& \left.a_{s}<\frac{\mathrm{S}_{n}\left(\sum_{\nu=1}^{s} t_{n}^{(\nu)}\right)-\mathrm{S}_{n}\left(\sum_{\nu=1}^{s-1} t_{n}^{(\nu)}\right)}{\left(n \rho \sigma t_{n}^{(s)}\right)^{1 / 2}}<b_{s}\right\}
\end{aligned}
$$

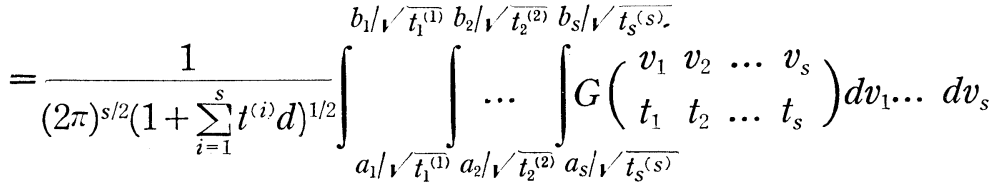

where

$$
G\left(\begin{array}{llll}
v_{1} & v_{2} & \ldots & v_{s} \\
t_{1} & t_{2} & \ldots & t_{s}
\end{array}\right)
$$

(III. II) $=\exp \left\{-\frac{v_{1}^{2}}{2\left(1+t^{(1)} d\right)}\right.$

$$
\left.-\frac{1}{2} \sum_{k=2}^{s} \frac{1+\sum_{\nu=1}^{k-1} t^{(\nu)} d}{1+\sum_{\nu=1}^{k} t^{(\nu)} d}\left(v_{k}-\frac{\sqrt{t^{(k)}} d \sum_{i=1}^{k-1} \sqrt{t^{(2)}} v_{i}}{1+\sum_{i=1}^{k-1} t^{(i)} d}\right)^{2}\right\},
$$

provided that following $\left(2.23^{\prime}\right)$ holds 


$$
t_{n}^{(\nu)}=\frac{m_{n}^{(\nu)}}{n}=t^{(\nu)}+o(1) \quad(\nu=1,2, \ldots, s)
$$

§3. The w. c. g. Stochastic Process. We now come to a position to introduce the following

Definition 4. Let $\Omega^{*}$ be the space of all functions $\mathrm{X}(t)$ defined over $0 \leqq t<\infty$. A stochastic process $\left({ }^{3}\right) \Omega$, a subset of $Q^{*}$, is said to be weakly contagious in the gaussian type, abbreviated: w. c.g., if it fulfills the following condition:

$(G)$ : Let $k$ be an arbitrarily assigned positive integer, let $0 \leqq$ $s^{(1)}<t^{(1)} \leqq s^{(2)}<t^{(2)} \leqq s^{(3)}<t^{(3)} \leqq \ldots \leqq s^{(k)}<t^{(k)}$ be arbitrarily assigned non-negative numbers, and further let $-\infty \leqq a_{i}<b_{i} \leqq \infty(i=1,2$, $3, \ldots, k)$ be couples of arbitrarily assigned real numbers. Then the probability of the elements $Q$-satisfying all of the conditions:

$$
a_{i}<X\left(t^{(1)}\right)-X\left(s^{(i)}\right)<b_{i} \quad(i=1,2,3, \ldots, k)
$$

is equal to

$$
\begin{aligned}
& \frac{1}{(2 \pi)^{s / 2}\left(1+\sum_{i=1}^{s} t^{(i)} d\right)^{1 / 2}}
\end{aligned}
$$

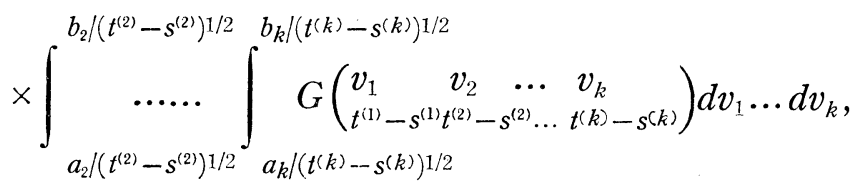

where the function $G\left(\begin{array}{llll}v_{1} & v_{2} & \ldots & v_{k} \\ \tau_{1} & \tau_{2} & \ldots & \tau_{k}\end{array}\right)$ is that defined in (III. II).

Putting now $J_{i}=\left(a_{i}, b_{i}\right), J^{(\infty)}=(-\infty, \infty), \quad I_{i}=\left(s^{(i)}, t^{(i)}\right)$ and $I_{i}^{\prime}=\left(t^{(i)}, s^{(i+1)}\right), I_{0}^{\prime}=\left(0, s_{0}\right)$, we shall denote the probability that all of the events (3.01) will happen by

$$
\operatorname{Pr}\left\{\begin{array}{llll}
J_{1} & J_{2} & \ldots & J_{s} \\
I_{1} & I_{2} & \ldots & I_{s}
\end{array}\right\}
$$

We shall then observe readily

Theorem 1. The w. c.g. stochastic process possesses the following properties:

$$
\operatorname{Pr}\left\{\begin{array}{llll}
J_{1} & J_{2} & \ldots & J_{s} \\
I_{1} & I_{2} & \ldots & I_{s}
\end{array}\right\}=\operatorname{Pr} .\left\{\begin{array}{llllllllll}
J^{(\infty)} & J_{1} & J^{(\infty)} & J_{2} & J^{(\infty)} & \ldots & J_{s-1} & J^{(\infty)} & J_{s} \\
I_{0}^{\prime} & I_{1} & I_{1}^{\prime} & I_{2} & I_{2}^{\prime} & \ldots & I_{s-1} & I_{s-1}^{\prime} & I_{s}
\end{array}\right\}
$$

(3) For the definition of the stochastic process, see the following paper:

J. L. Dоов: Stochastic processes depending on a continuous parameter, Trans. Amer. Math. Soc., 42 (1937), 107-140. 
( V)

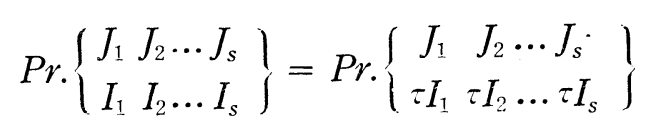

for any positive number $\tau$, where $\tau I_{j}$ means the interval which consists of the $\tau$-translated one of the interval $I_{j}=\left(s^{(j)}, t^{(j)}\right)$, that is, $\tau I_{j}=\left(\tau+s^{(j)}, \tau+t^{(j)}\right)$.

$\S 4$. The Mean-Value, Standard Deviation and Autocorrelation Coefficients of the w. c. g. Stochastic Process. Let us adopt, as usually, the convention that

$$
E\left\{\left(X\left(t^{(1)}\right)-X\left(s^{(1)}\right)\right) n_{1}\left(X\left(t^{(2)}\right)-X\left(s^{(2)}\right)\right) n_{2} \ldots\left(X\left(t^{(k)}\right)-X\left(s^{(k)}\right)\right) n_{k}\right\}
$$

means

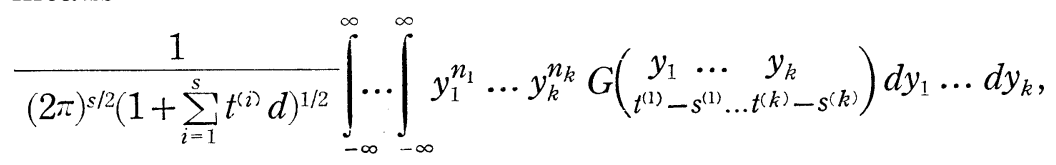

for any assigned non-negative integers $n_{1}, n_{2}, n_{3}, \ldots, n_{k-1}, n_{k}$ and for any assigned $2 k$ real numbers such that

$$
0<s^{(1)}<t^{(1)} \leqq s^{(2)}<t^{(2)} \leqq s^{(3)}<t^{(3)}<\ldots \leqq s^{(k)}<t^{(k)} .
$$

There is no difficulty in calculating these values, which will give some insight to our stochastic process. We shall restrict ourselves to enunciating

Theorem 2. The mean value and the standard deviation of the w.c.g. stochastic process in the intervals $(s, t)$, where $0<s<t$ $<\infty$, is given by

$$
\begin{aligned}
& E\{(X(t)-X(s))\}=0 \\
& \sigma\left\{(X(t)-X(s))^{2}\right\}=(t-s)[1+(t-s) d],
\end{aligned}
$$

and the autocorrelation coefficient between $X\left(t^{(1)}\right)-X\left(s^{(1)}\right)$ and $X\left(t^{(2)}\right)-X\left(s^{(2)}\right)$, which we denote by $R\left(\begin{array}{c|c}s_{1} & s_{2} \\ t_{1} & t_{2}\end{array}\right)$, is given by

$$
R\left(\begin{array}{c|c}
s_{1} & s_{2} \\
t_{1} & s_{2}
\end{array}\right)=\frac{\left(t_{1}-s_{1}\right)\left(t_{2}-s_{2}\right) d}{\left[\left(t_{1}-s_{1}\right)\left\{1+\left(t_{1}-s_{1}\right) d\right\}\left(t_{2}-s_{2}\right)\left\{1+\left(t_{2}-s_{2}\right) d\right\}\right]^{1 / 2}},
$$

provided that

$$
0<s_{1}<t_{1} \leqq s_{2}<t_{2} .
$$

Theorem 2 may be proved either by the direct calculation of the formula (4.01) or by considering the limiting values of the formulae, established by PóLYA, concerning the corresponding 
statistical quantities in the original PólYa-EgGenberger's scheme. It may be not perhaps without interest to remark that

$$
\begin{aligned}
R\left(\begin{array}{c|c}
s_{1} & s_{1} \\
t_{1} & t_{2}
\end{array}\right) & =\frac{\left(t_{1}-s_{1}\right)\left\{\left(t_{2}-s_{1}\right) d+1\right\}}{\left[\left(t_{1}-s_{1}\right)\left\{1+\left(t_{1}-s_{1}\right) d\right\}\left(t_{2}-s_{1}\right)\left\{1+\left(t_{2}-s_{1}\right) d\right\}\right]^{1 / 2}} \\
& =\left(\frac{d+\frac{1}{t_{2}-s_{1}}}{d+\frac{1}{t_{1}-s_{1}}}\right)^{1 / 2},
\end{aligned}
$$

provided that $0<s_{1}<t_{1}<t_{2}$. 